Decl. $+8 \mathrm{I}^{\circ} 10^{\prime \prime} 5$. The star is No. $113-4$ of Fedorenko's Catalogue.

The following are times of minima of $U$ Cephei to the end of April, inferred by Mr. Knott from his observations on March I8 :-

$$
\text { h. m. h. m. }
$$

March 23 ... I2 o G.M.T. A pril I2 ... I0 37 G.M.T.

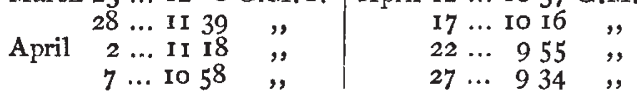

\section{THE CURRENT METER OF PROF. A. R. HARLACHER ${ }^{1}$}

PROF. HARLACHER, of the Technical High School at

Prague, was the first to construct a current meter which obviates all the difficulties and drawbacks of the instruments

\section{FIG. 1.}

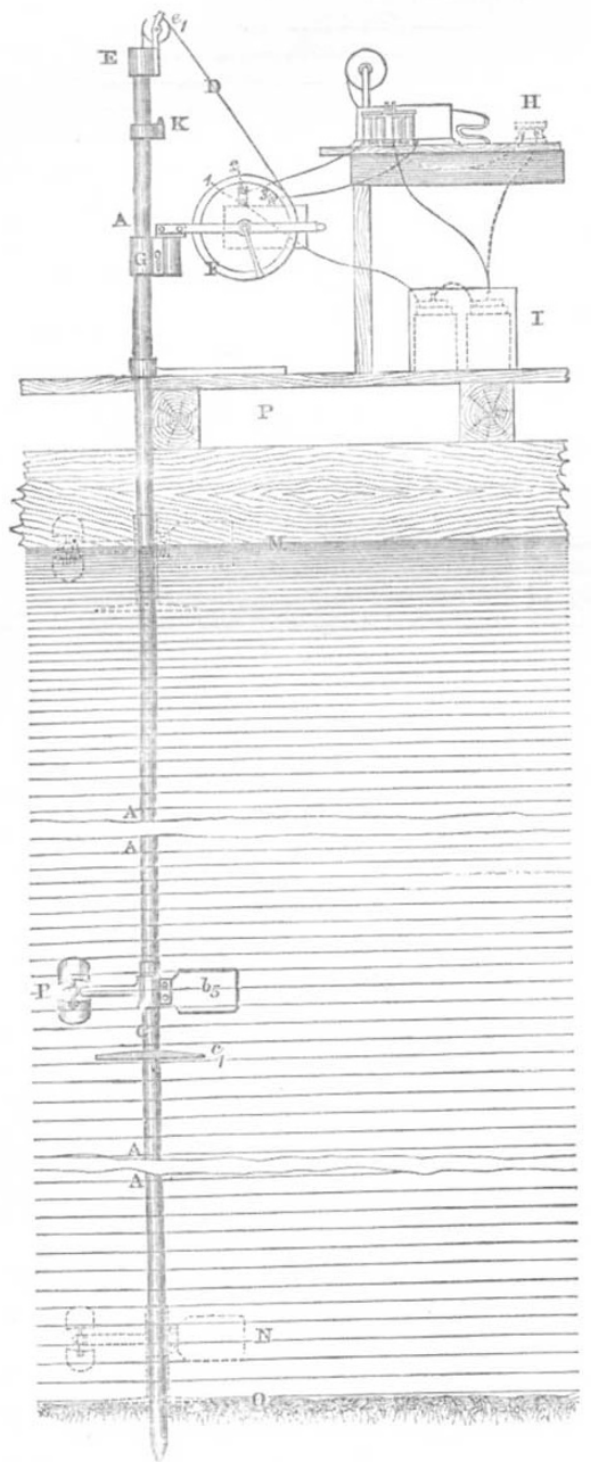

previously employed. The Harlacher current meter permits the velocity to be determined in the shortest possible time.

It is unnecessary to describe all the stages in the invention of the present form of the Harlacher meter. It is sufficient to say

${ }^{x}$ By Richard Blum, City Engineer, Leipzig. From advanced copy of a paper in the Proceedings of the Institution of Civil Engineers, by permission of the Council. that Prof. Harlacher worked for several years at its improve. ment, and that his success was acknowledged by the award, at the Paris Exhibition, of two gold medals.

The Harlacher meter is constructed as follows:- For the movable staff, on which the Woltmann meter is fixed, an immovable staff or rod is substituted, which is planted firmly in the bed of the river, and along which the meter slides up and down during the observations on any one vertical. This rod is a cast-iron tube, with a solid point at the lower end, A, A (Fig. FIG. 2.

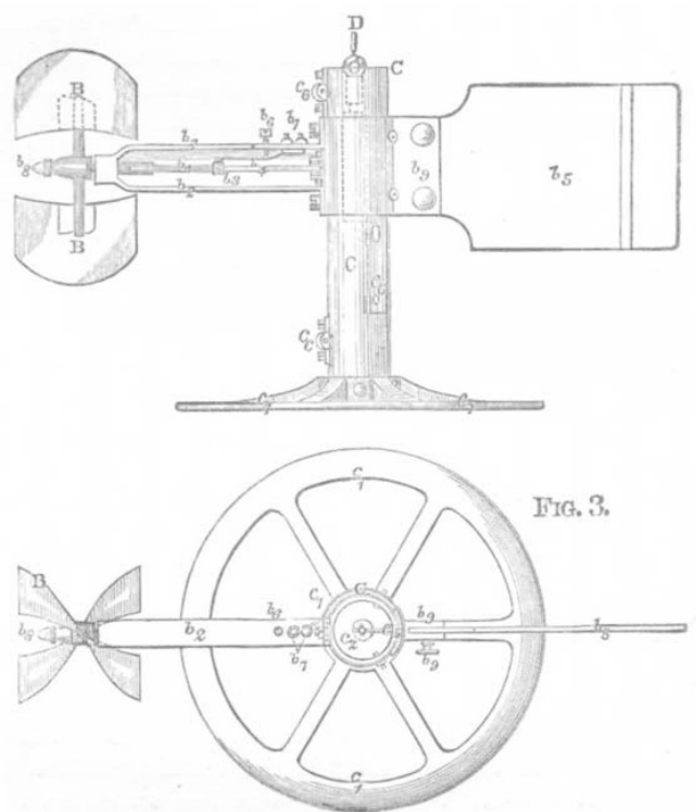

I). The other parts of the apparatus, except the electric battery and indicator, are fastened to the tube, so that the whole can be moved from one vertical to another, without having to be taken apart. The screw of the meter, $\mathrm{B}$, is two-bladed. For very small velocities, it would be preferable to adopt a fourbladed screw of larger diameter. The screw is fixed on a steel shaft, $b$ (Fig. 2), which has an eccentric enlargement at one point, $b_{3}$. This makes contact with the steel spring, $b_{4}$, at each revolution of the meter. These contacts complete the electric circuit, and the current which passes actuates the electric clock

Fig. 5.

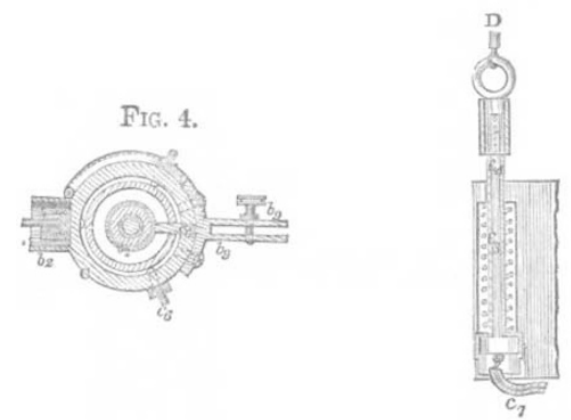

or indicator. The weight of the screw $\mathrm{B}$, the shaft $b$, and the brass box $b_{2}$, which carries the shaft, is balanced by a counter. weight $b_{5}$ (Figs. 1,2 , and 3 ). This keeps the axis of the instrument in a perfectly horizontal position. The screw, $b_{6}$, serves to regulate the pressure of the spring, $b_{4}$, while the two screws, $b_{7}$, fasten the spring to the brass frame which surrounds and protects the shaft. The shaft is square at the end, which receives the screw, which is put on and held fast by a nut, $b_{8}$ (Figs. 2 and 3). The brass frame, $b_{2}$, is fixed to a hollow cylinder, c. Below the hollow cylinder, $c$, is a plate, $C_{1}$ (Figs. 2 and 3 ), which pre- 
vents the instrument approaching too closely to the bed of the river, where it might be injured or retarded by obstacles. In the interior of the cylinder, $\mathrm{C}$, there is a cylindric case, $c_{2}$ (Figs. 3 , 4, and 5), in which a brass spring is fastened, and through which the pin, $c_{3}$, is carried. To this pin the end of the sus pending rope, $\mathrm{D}$, is fastened. The internal diameter of the cylinder, $\mathrm{C}$, is a little larger than the outside diameter of the hollow rod, $\mathrm{A}$, on which it is to slide. The part, $c_{2}$, to which the rope is attached, is connected with $\mathrm{C}$ by an arm which passes through a vertical slit in the hollow rod, A. Thus, the instrument is kept always, if the pipe, A, is properly placed, with its axis normal to the plane of the cross section. The cylinder, $\mathrm{C}$, is also fitted with rollers, $c_{6} c_{6}$, which render the motion on the fixed rod easy. After the instrument has been placed on the rod or staff, a bracket, E (Fig. 1), with a pulley, $e_{1}$, is attached at the top, and the rope is carried over this pulley. The rope, $\mathrm{D}$, is wound on a barrel, $\mathrm{F}$. This barrel is fixed with the frame, $f_{1}$, and the pin, $f_{2}$, on the arm, G (Figs. I, 6, and 7 ) which is firmly fastened to the hollow rod, A. With the barre is connected the apparatus, $f_{3}$, registering the depth at which the meter is at any moment. Tbe fan, $f_{4}$, and gearing, $f_{5}$, regulate the rate of rotation of the barrel and permit the adjustment of the speed of the meter in its descent along the rod, A. By the handle, $f_{6}$, the meter is again raised. The lever, $f_{7}$, and ratchet

FIG. 6.

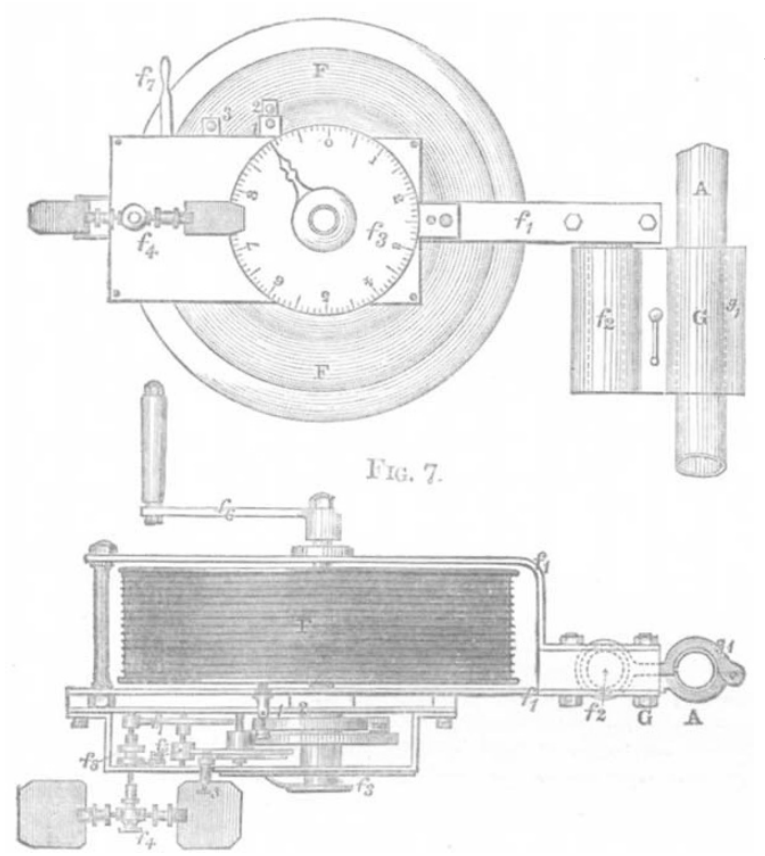

wheel, $f_{8}$ (Fig. 6), arrest the rotation of the barrel. The movement begins tas soon as the ratchet is lifted by the lever. On the frame of the barrel, $F$, are fastened the contact screws, I, 2, 3 (Figs. I, 6 and 7), for attaching the wires of the electric circuit. The screw, I, is connected with the rope, D, which is a copperwire rope covered with insulating material. The rope is in electric contact with the shaft of the screw through the spring, $c_{3}$ (Fig. 5), because an insulated wire, $c_{7}$ (Figs. 5 and 3 ), connects the lower end of the pin, $c_{3}$, and the loop of one of the screws, $b_{7}$ (Figs. 2 and 3 ), which fasten the spring to the brass frame, $b_{2}$. The other conductor is the cast-iron pipe, $\mathrm{A}$, which is in contact with the rest of the apparatus through the parts C, G, $f_{1}, f_{2}$ and F (Figs, 6 and 7). These parts are connected with the screw 2 (Figs. 1, 6, and 7). By putting a wire into the loop of screw 3 the depth of the meter below the water-line can be registered electrically. The registering apparatus, $\mathrm{H}$ (Fig. I), has two dials, one marking single revolutions and the other hundreds of revolutions.

If desired, a recording arrangement can be added, the rotations of the meter being marked on a slip of paper in the same way as in a writing telegraph or chronograph. Prof. Harlacher used this arrangement in determining the variation of velocity at a given fixed point. The battery, I, and the clock, or indicator, $\mathbf{H}$, with the rod, A, carrying the meter, are placed on a float, $\mathbf{P}$. The sight vane, $\mathrm{K}$, is fastened to the rod, $\mathrm{A}$, so that it is parallel to the plane of the cross section, and then the axis of the screw is normal to the cross section and parallel to the current. The float is anchored in large rivers and fastened to guide ropes or poles in smaller streams. As soon as the work at one vertical of the cross section is finished, the anchor ropes on one side are slackened and on the other tightened, so as to bring the float into a new position in an easy and a speedy manner. The float must be built so as to be capable of supporting four or five persons.

The determination of the mean velocity at one vertical, by allowing the meter to slide once from the surface of the stream to the bottom, is accomplished thus. The meter, B, and all its connections, c, $c_{1}, \&$ c., are brought to within a few inches of the water surface, the fingers of the electric clock being set to zero. Then the barrel, $F$, is released by the lever, $f_{7}$, Fig. 6 . As soon as the axis of the screw touches the water surface a signal is given, the electric clock is brought into the circuit by a spring lever, and begins to count the rotations of the screw. It is necessary to commence with the meter some small distance above the water surface, in order that it may acquire the proper descending velocity previous to the counting of the rotations. In a certain number of seconds the meter descends from $\mathrm{M}$ to $\mathrm{N}$ (Fig. r), having at each point in its descent acquired the velocity of rotation corresponding to the velocity of the water at that depth. Dividing the number of revolutions by the number of seconds the rate of rotation corresponding to the mean velocity at that vertical is found. The fact that the disk, $c_{1}$ (Figs. I, 2, and 3), prevents the meter from descending exactly to the bottom entails a small correction. This correction, however, will be more insignificant the larger the difference of the heights $M N$ and $N O$, that is, the deeper the river in which the observations are made. It is a matter of course that the readings of the instrument at each vertical should be repeated, and the average of the results taken for the true mean velocity. The results of single measurements will not differ much from each other, but the repetition of the reading will give a certainty that all the variations of the velocity at the given vertical are allowed for.

Before using the meter, its constants must be determined in the same manner as with the Woltmann apparatus. A length is marked out in a still.water basin, and the meter is frequently moved through this distance at different speeds. It is essential that the movement of the boat or float on which the meter is fixed should be a uniform one.

The above description of the apparatus will prove that the advantages of this form of meter are of considerable importance.

\section{THE STORAGE OF ENERGY $Y^{1}$}

$T H E$ subject of this lecture has been called by the world at large, even by well-informed Punch, "The Storage of Force." Why, then, have I ventured, in my title, to differ from so popular an authority? For this simple reason-that you cannot store force any more than you can store time. There is as much difference between force and work, as there is between a mile and the speed of a train or between a ship and a voyage. Work involves two distinct ideas combined, whereas force only involves one. When a weight rests on the ground, the weight pushes the ground down with a certain force, and the ground pushes the weight up with the same force. If, then, there were such a thing as a storage of force, the mere resting of a weight on the ground would be such a storage, since the force exerted between the weight and the ground never grows less. But, I need hardly say, it would be beyond the ability of the cleverest engineer to work a machine, or drive a train, by using a weight resting on the ground ; the very expression, "dead weight," shows how useless it is for the practical purposes of producing motion. A weight resting on the safety-valve of a steam-engine may be a very good means of adjusting the pressure at which the valve shall open and liberate the excess steam, but this weight will never work the engine.

Work is force exerted through space; if a weight $\mathrm{P}$ be raised through $F$ feet, $P \times F$ foot-pounds of work will be done, and there will be a store of $\mathrm{P} \times \mathrm{F}$ foot-pounds of work in the raised weight.

The continuous evaporation of the water from the seas and

Abstract of a lecture delivered at the London Institution on Thursday, March 2, by Prof, W. E. Ayrton, F.R.S. 